\title{
A atenção domiciliar no processo de estabelecimento da saúde bucal na estratégia da saúde da família
}

\author{
Home care in the process of establishing oral health in the family health strategy
}
El cuidado domiciliario en el proceso de establecimiento de la salud bucal en la estrategia de salud familiar

José Milton de Aquino e Silva Neto ${ }^{1 *}$, Maxsuel Fabian Cavalcante Silva ${ }^{1}$, Anderson dos Santos Panaro ${ }^{1}$, Altamiro Júnior Paranhos Cesar de Mendonça ${ }^{1}$, Michelle Leão Bittencourt Brandão Medeiros $^{1}$, Tayguara Cerqueira Cavalcanti ${ }^{1}$.

\section{RESUMO}

Objetivo: Efetuar uma revisão narrativa para explorar as concepções e forma de atuação dos Cirurgiões Dentistas (CD) nos cuidados realizados na cavidade bucal na atenção domiciliar (AD) na estratégia da saúde da família (ESF). Revisão Bibliográfica: A AD vem se desenvolvendo cada vez mais, buscando sempre o bem-estar do paciente através do atendimento humanizado, o CD faz parte da integralidade dessa equipe multiprofissional que compõe a ESF, dos quais buscam promover com aspectos primordiais o estabelecimento da saúde oral, principalmente dos pacientes que não possuem locomoção, dispondo como seguimentos aguardados o estabelecimento de prevenções e tratamento de possíveis patologias, visando por sua vez a restauração do indivíduo no $A D$, assegurando aos cuidados terapêuticos necessários. $O C D$ retém sua indispensabilidade, visto que apresentam funções qualificadas em executar tratamentos, com intuito de oferecer cuidados referente a saúde oral, instrução e motivação. Considerações Finais: À vista disto, os CD's nas visitas domiciliares encarregam-se de um fundamental papel, em que proporciona um conforto de maneira física e social dos pacientes que são incapacitados por diversas maneiras de irem até Unidade de atendimento.

Palavras-chave: Saúde bucal, Clínicas odontológicas, Saúde da família, Visita domiciliar.

\section{ABSTRACT}

Objective: Carry out a narrative review to explore the conceptions and way of performance of Dental Surgeons $(C D)$ in the care performed in the oral cavity in home care (AD) in the family health strategy (ESF). Literature Review: The AD has been developing more and more, always seeking the well-being of the patient through humanized care, the $C D$ is part of the integrality of this multiprofessional team that makes up the FHS, of which they seek to promote the establishment of oral health with essential aspects, mainly of patients who do not have locomotion, having as expected follow-up the establishment of preventions and treatment of possible pathologies, aiming in turn to restore the individual in the $A D$, ensuring the necessary therapeutic care. The $C D$ retains its indispensability, since they have qualified functions in carrying out treatments, in order to offer care regarding oral health, instruction and motivation. Final Considerations: In view of this, the DC's in home visits are responsible for a fundamental role, providing physical and social comfort to patients who are disabled by different ways of going to the care unit.

Keywords: Oral health, Dental clinics, Family health, House calls.

\section{RESUMEN}

Objetivo: Realizar una revisión narrativa para explorar las concepciones y forma de actuación de los Cirujanos Dentales (CD) en el cuidado realizado en la cavidad bucal en la atención domiciliaria $(A D)$ en la estrategia de salud familiar (ESF). Revisión Bibliográfica: La AD se ha ido desarrollando cada vez más,

${ }^{1}$ Centro Universitário CESMAC (CESMAC), Maceió - AL. *E-mail: milton_neto_166@hotmail.com 
buscando siempre el bienestar del paciente a través del cuidado humanizado, la CD forma parte de la integralidad de este equipo multiprofesional que compone la ESF, del cual buscan promover el establecimiento de la salud bucal con aspectos esenciales, principalmente de los pacientes que no tienen locomoción, teniendo como seguimiento esperado el establecimiento de prevenciones y tratamiento de posibles patologías, con el objetivo a su vez de restaurar al individuo en la $A D$, asegurando los cuidados terapéuticos necesarios. La CD conserva su indispensabilidad, ya que tiene funciones calificadas en la realización de tratamientos, con el fin de brindar cuidados en materia de salud bucal, instrucción y motivación.

Consideraciones Finales: Ante esto, los CD's en visitas domiciliarias tienen un rol fundamental, brindando confort físico y social a los pacientes que se encuentran discapacitados por las diferentes formas de acudir a la unidad de cuidados.

Palabras Clave: Salud bucal, Clínicas odontológicas, Salud de la familia, Visita domiciliaria.

\section{INTRODUÇÃO}

O crescimento e o paralelismo, tornou-se uma instigação ao que se refere a Saúde Pública no Brasil, seu processo de renovação se deu com a formação do Sistema Único de Saúde (SUS), em que ocorreu através da Constituição Federal de 1988. O insucesso referente ao exemplar do assistencialismo referente as condutas terapêuticas, relevantes até o instante, proporcionou modificações contingenciais principalmente nas condutas de políticas públicas na área da saúde, estabelecendo por sua vez alterações nos processos de serviços na saúde (DE-CARLI AD, et al., 2015).

No final da década de 90, buscando por sua vez obter a consolidação do SUS, o Ministério da Saúde expôs um novo planejamento, destacando o Programa de Saúde da Família (PSF), dispondo assim com a atenção básica, tendo como foco a realização do primeiro acesso do usuário ao sistema de saúde. Traçando em demarcações de áreas referente a forma de atividade de cada profissional, sendo feito esquematizações estruturais da região adjunta, o PSF possui como finalidade estabelecer intervenções fundamentadas em parâmetros que apresentem perigo da integridade à saúde, expandindo condutas a atenção familiar.

Hoje em dia, o PSF ficou sendo estabelecido como sendo uma Estratégia de Saúde da Família (ESF), o que antes era um programa em que possuía início e fim. Enquanto a Estratégia Saúde da Família é definida como algo contínuo em que busca uma remodelação da atenção primária dos quais dispõe de intervenções elaboradas e pareceres periódicos (BIZERRIL DO, et al., 2015).

A Estratégia de Saúde da Família demonstrou uma mudança enfatizando o foco de atuação atentando mais para o bem-estar familiar, sendo acompanhada no seu ambiente social em que compreende o segmento populacional determinado, com atualização contínua, respeitando suas especificidades, por diversos assistentes multiprofissionais, planejando dessa maneira determinadas condutas mais inclusivas, possuindo cuidados epidemiológicos dos quais são fatores condicionantes e determinantes, nas populações humanas. Como alicerces organizativos com ações de maneira coletiva (RODRIGUES FFL, et al., 2012).

A princípio os profissionais da Odontologia não foram integrados nesse início, apresentando uma incoerência, pois recomenda-se intervenções de maneira preventiva, possibilitando promoções, processos terapêuticos e restauração em saúde, porém não inseriu promoções da cavidade oral, demonstrando como se não constituísse parcela da saúde de maneira integral, agindo de maneira particularizada quando comparada aos outros serviços prestados na saúde (MOURA MS, et al., 2013; BIZERRIL DO, et al., 2015).

Com a composição dos CD's na constituição da equipe multiprofissional, de maneira a integrar na saúde da família, a saúde da cavidade oral obteve frutos positivos dos quais foram estabelecidas intervenções nas comunidades, tal qual, que colaboraram para um melhor desenvolvimento referente à atenção básica e, alcançou a identificação de como é importante para o processo de humanização unidos ao Ministério da Saúde, em que divulgou na Portaria MS/GM no 1.444 de 2000, (BRASIL, 2000). Onde determina estímulos de verbas para a remodelação da maneira a ser tratada a saúde da cavidade bucal, por meio da Estratégia da saúde da família (SILVESTRE JAC, et al., 2013). 
Um dos grandes avanços significativos referente aos instrumentos de atuação estabelecida pela Equipe das quais integram a ESF é a visita domiciliar (VD), dos quais houve consentimento do estabelecimento de conexões entre o profissional e o paciente, conhecendo e compreendendo a vivência da determinada região trabalhada e perceber a dinâmica situacional dos vínculos e realidades que refere-se as famílias (DE-CARLI $A D$, et al., 2015).

Do mesmo modo existe a evolução de ações de promoção da saúde, em que trata como determinada importância medidas preventivas de agravamentos e retém à atenção na saúde, sendo feito o acolhimento de todo parentesco familiar, sendo levado em consideração as indispensabilidades de atuações estabelecidas pelo multiprofissionalismo. Na área que abrange a atuação da equipe de saúde, o posicionamento, as condutas de planejamentos, execuções e a efetivação das atividades necessitam serem feitas pelas equipes de saúde que estão presentes, para só assim organizar os dados e analise situacional da respectiva área (REIS WG, et al., 2015).

As diretrizes que correspondem a forma de atuação na Atenção Básica $n^{\circ}$ 17, dos quais norteiam a padronização do estabelecimento da atenção à saúde da cavidade oral no Sistema Único de Saúde, evidencia que a constituição referente a atenção domiciliar (AD) são imprescindíveis, no entanto são encontrados certas barreiras ao que se expõe a Estratégia da saúde Básica (BRASIL, 2006). A AD relacionada a saúde bucal compreende de forma direta os acompanhamentos domiciliares aos usuários do SUS, em que necessitam de atenções básicas, podendo-se destacar a forma de abordagem no ato do atendimento, levando em consideração a realidade do paciente e as condutas de internações feitas em sua própria casa, detendo cada profissional a responsabilidade dos cuidados e atenções necessárias (REIS WG, et al., 2015).

A AD vem sendo definida em conformidade como uma área de seriedade em que as equipes de saúde envolvidas visam sempre o bem-estar do paciente como um todo, contudo os profissionais da saúde encontram-se com vários obstáculos, ao ser levado em consideração as adversidades de estabelecer a esses pacientes uma atenção de maneira integra nos domicílios e concomitantemente avançar com os cuidados terapêuticos propícios, não só para o indivíduo que de fato tem a necessidade, como também para todos os membros constituintes da respectiva família (KOBAYASHI HM, et al., 2015).

É notório e observado que os atendimentos domiciliares são direcionados a pessoas que não possuem mobilidades, dos quais encontram-se em situações muito das vezes acamados, pacientes que apresentam gestações de riscos e pacientes com necessidades especiais, dessa maneira a atenção domiciliar é uma forma de escolha dos quais ocorre a atenção dos paciente deem um local desinstitucionalizado de pessoas que apresentam históricos de quadros internativos em ambientes hospitalares e podem ser acompanhados em casa (FERRAZ GA e LEITE ISG. 2016).

À vista do que foi mencionado, este estudo dispõe como um dos principais objetivos executar uma revisão narrativa por meio de leitura de artigos mais relevantes sobre a temática em que avaliam a relevância do desempenho de toda equipe que integra a saúde da cavidade oral na conjuntura da assistência domiciliar no ciclo da ESF.

\section{REVISÃO BIBLIOGRÁFICA}

\section{$O$ atendimento domiciliar aos pacientes debilitados}

Silva RM, et al. (2018); demonstram em estudos feitos que o paciente têm que ser examinado de forma completa, levando em consideração os cuidados da cavidade oral, visando levar em consideração a existência do rastreamento de possíveis doenças sistêmicas dos quais a equipe multiprofissional é responsabilizada pela restauração psicossocial do paciente que está sendo acompanhado, quanto de todos os membros da família, além disto a equipe deve se comprometer em fornecer orientações referente ao processo de autonomia para os paciente debilitados e familiares.

A atenção domiciliar é composta por meio de estratégias que visam pôr em pratica atividades das quais estabelecem a como prioridade os cuidados à saúde, possuindo como frutos aguardados a prevenção e os cuidados em estágio inicial da patologia, possuindo uma ótica na prevenção, promoção em que estabelece a restauração do paciente no AD, procurando confirmar a continuidade das atenções (PINTO VG, 2013). 


\section{Atuação do cirurgião dentista no AD}

Do mesmo modo em que outros profissionais como exemplo médicos, enfermeiros, psicólogos, entre outros, o CD na esfera em que representada o planejamento da saúde da família possui seu grande valor, como pode ser analisado nos direitos das competências em efetuar visitas em domicílio tendo como intuito oferecer atenção em toda cavidade oral, buscando fazer anamneses e conhecer um pouco do cotidiano não só do paciente como de todos os membros que estão presentes naquele grupo (SILVA RM, 2016).

Para poder ser elaborado e organizado uma forma de atendimento domiciliar planejado e com bons resultados, os profissionais possuem como critérios oferecer o exercício de maneira continua, analisando desde a primeira visita até a última, tentando dessa forma observar a evolução do paciente, tendo em vista que essa metodologia não pode ser estabelecidas de maneiras isoladas, sendo levado em consideração o diagnóstico final e prognóstico do tratamento do paciente, além do acompanhamento periódico para a continuidade da integridade da saúde da cavidade bucal do paciente assistido (PINTO VG, 2013; SILVA RM, 2016).

Nesse enquadramento, as principais etapas de uma boa programação de planejamento são: estabelecer uma boa anamnese de forma detalhada; ter em vista como é o cotidiano do paciente e os cuidados que os cuidadores e/ou familiares possuem, organização e continuidade no tratamento proposto, buscar de maneira humanizada entender a realidade do mesmo e estabelecer um diálogo em que ele possa entender a importância dos cuidados que estão sendo propostos (SALAS MM, et al., 2015).

Para que os Cirurgiões Dentistas estejam vinculados a essa equipe de saúde é necessário saberem as diretrizes que regulamentam as definições básicas ao que se refere aos princípios éticos que envolvem o SUS, tendo os mesmo que estarem aptos e qualificados, sabendo as maneiras de atuações que necessitam serem cumpridas ao fazer o atendimento domiciliar, buscando sempre conhecer o paciente na hora da anameses, buscando sempre ter atenção em cada resposta do paciente.

Para isso é importante fazer de maneira individualizada, buscando saber o histórico de eventuais doenças da cavidade oral que o mesmo já possuiu, sempre prezando pelo acolhimento e atendimento humanizado para só assim o paciente dispor da integralidade, equidade, e o acolhimento. Tais maneiras de atuação faz com quer o paciente crie confiança e um vínculo com o CD (SILVA RM, et al., 2018).

\section{Importância do atendimento domiciliar}

Um dos principais pontos relevantes para o estabelecimento da atenção domiciliar além de dar mais atenção e conforto ao paciente que está passando por tratamentos terapêuticos é estabelecer a "desospitalização" dos quais proporciona celeridade no processo de alta hospitalar com cuidado continuado do paciente em sua própria residência, fazendo com quer o profissional de saúde possua uma interrelação melhor com o paciente, sendo indicado como um componente em que se faz as modificações totais referentes aos serviços de saúde prestados.

Visto que os serviços te atenção básica são oferecidos para todos os integrantes que constituem a região estabelecida, nas distintas moradias, buscando assim instituir, evidenciar ou até mesmo recompor a saúde; potencializando um bom grau de independência, minimizando assim decorrências de eventuais enfermidades ou até mesmo aquisição de infecções que poderiam serem adquiridas no ambiente hospitalar, visto que a maioria desses pacientes são bastante debilitados, possuindo o sistema imunológico baixo (REIS WG, et al., 2015).

A maneira de executar um atendimento domiciliar dispõe de uma característica bastante particularizada e extensiva, à vista disso, usa-se muito na área político-administrativa em que possibilita uma hierarquização dos quais facilita a administração do território trabalhado pelos profissionais de saúde, podendo ser tanto de forma pública quanto privada. Contudo, mesmo sabendo-se que existem definições especificas no ato da idealização dos projetos das quais possuem inúmeras maneiras de compreensões, apresentam-se como sendo uma metodologia que fundamenta intervenções sociais por intermédio do estabelecimento da compreensão de inúmeras possibilidades que analisam o desenvolvimento e a integridade, pretendendo restabelecer impasses e buscar entender às obrigações estabelecidas de forma coletiva ou individual da sociedade (MENICUCCI TMG, 2009; PINTO VG, 2013). 
O AD é de suma importância ao que se refere aos processos de ferramentas na hora de executar a instrumentalização do amparo em domínio pelos membros que constituem a Estratégia de saúde da família (ESF), atendendo aos conceitos da assistência continua, à dimensão em que diminui interrupções as atenções prestadas ao paciente quando se analisa a forma de mediações de familiaridade entre a equipe multiprofissional da Unidade de Atenção Primária a Saúde (UAPS) e os usuários, no seu respectivo lar (BARATIERI T e MARCON SS, 2011).

Rodrigues FFL, et al. (2012) define que as atenções domiciliares executadas pelos Cirurgiões Dentistas promovem intervenções de promoção continua de saúde bucal e por consequência previne à saúde dos indivíduos que estão sendo cuidados, tendo em vista que se constata a grande indispensabilidade de recursos terapêuticos que envolvem os tratamentos odontológicos, dos quais são feitos de maneiras adequadas 0 assistencialismo. Desse modo, a construção de fichas cadastrais específicas, dispondo tanto do cadastro individualizado quanto familiar, possibilitando assim o atendimento bem mais elaborado, visando a independência do indivíduo e a remediabilidade em que se retrata.

O processo de assistencialismo estabelecido de forma domiciliar busca complementar as necessidades dos pacientes que encontram-se incapacitados, temporariamente ou de maneira permanente de buscarem as Unidades de pronto Atendimentos, esses grupos de pacientes que são acompanhados pelos profissionais não apresentam doenças especificas e a idade varia muito. Suas designações são fundamentadas através de avaliações do grau de risco que o paciente venha a possuir, por intermédio de classificação de evidenciações a elementos que venham a prejudicar à saúde, levando em consideração os possíveis aumentos de desordem estabelecida pela tríade: problemas físicos, psicológicos e sociais.

Inúmeros grupos de pacientes apresentam esses riscos e que devem ser acompanhados com bastante atenção, porém os que mais são prevalentes nos cuidados domiciliares, correspondem os pacientes com Necessidades Especiais (PNEs), os debilitados e estão acamados e Puérperas /Recém-nascidos (COELHO FLG e SAVASSI LCM, 2016).

\section{PNES no atendimento domiciliar}

Os indivíduos que apresentam alguma forma de obtenção de atenção especial na maioria das vezes são pacientes que demonstram possuírem determinadas limitações ou até mesmo variações físicas causada por alguma doença sistêmica ou congênita, podendo ser classificada com um grau menos elevado, diagnosticada como simples ou até mesmo com maior complexibilidade, de maneira breve onde se pode ver o quadro do paciente evoluir ou um quadro que demonstre a irreversibilidade do paciente, requerendo uma conduta de plano de atendimento de maneira diferenciada, ou seja, especializada, interdisciplinar e mantendo um procedimento padrão através do acompanhamento dos protocolos existentes (SILVA RM, et al., 2017).

Posto isto, o atendimento aos pacientes especiais é classificado como todo atendimento ao indivíduo que demonstra apresentar problemas em seus compostos biológicos, problemas que envolvem a parte psicossocial, dos quais apresentem determinada insuficiência ou inaptidão para a excursão de ações dentro dos exemplos que consideram habituais para a maioria dos indivíduos (DE-CARLI AD, et al., 2015; SILVA $\mathrm{RM}$, et al., 2017).

Para Pinto VG, (2013) os pacientes considerados como sendo portadores de necessidades especiais, são todos os indivíduos que demandam atenções especializadas, e os procedimentos terapêuticos utilizados pelos Cirurgiões Dentistas precisam serem estabelecidos de forma particularizada de um indivíduo para o outro, sendo analisado as limitações existenciais, relacionadas aparte emotiva, perceptiva associada a cognição ou psicológica com ênfase na parte comportamental. Sendo, deste modo, de grande relevância para - $C D$ e a equipe multiprofissional estabelecer um conhecimento prévio, visando a melhor forma de atendimento (BIZERRIL DO, et al., 2015).

Ainda que os pacientes especiais se queixem de sofrimentos provocados por dores relacionados a processos infecciosos da cavidade oral, e sabendo-se que essas infecções possuem potencialidade de intensificar as condições sistêmicas do usuário com problemas relacionados a sua deficiência, de modo infeliz a saúde oral continua sendo vista com pouca precedência quando contraposta as demais condutas de atenções médicas aplicadas a esses pacientes (BIZERRIL DO, et al., 2015). 
É de suma importância estabelecer o real estado de saúde na cavidade oral do paciente para não prejudicar ainda mais possíveis doenças sistemas que o mesmo possa ter, os Cirurgiões Dentistas devem trabalhar de forma conjunta com outros profissionais da saúde para poder fazer o direcionamento e avaliações, observando as características que possuem potencial de interferência no plano de tratamento. Deve ser observado com atenção a forma que o paciente se comporta, analisando os sintomas que os pacientes ressaltam ou os cuidadores e os sinais estabelecidos de forma clínica, desta maneira, é estabelecido com maior precisão e segurança o diagnóstico final, de acordo com Campos CC, et al., (2009).

\section{Atendimento domiciliar em pacientes acamados}

Paciente dos quais encontram-se acamado precisam de maior atenção de maneira domiciliar, visto que exibem insuficiências de locomoção e das habilidades funcionais que geram determinados tipos de obstáculos ao que se refere aos aspectos posturais e ao movimentos para a execução de trabalhos do cotidiano (SILVA $\mathrm{RM}$, et al., 2017). Silva RM, et al. (2018); demonstram que esses pacientes vivenciam determinadas debilidade não só física como psicológica, descrita por degenerações e problemas crônicos, resultante de inúmeras patologias, dos quais prejudicam de forma direta a plenitude tanto físicas quanto sociais, acarretando eventos que demandam os cuidados de terceiros por um grande período de tempo.

Pode-se observar que não existe uma faixa etária especifica para esse público alvo, muito menos para as doenças que mais acarretam a essa condição, porém o acompanhamento domiciliar é estabelecida através do nível de demanda e falta de capacidade do indivíduo, dando prioridade principalmente para as pessoas que apresentam maior vulnerabilidade. De acordo com o código de Assistência Domiciliar na Atenção Primária à Saúde Brasil (2006); os usuários do SUS que possuem patologias de forma crônica, dos quais precisam de terceiros para sua integridade física e locomotora, pacientes em fases moribunda, é necessário o AD dos profissionais de saúde com o objetivo de obter a integridade desse indivíduo.

Os CD's precisam ter atenção na hora do atendimento com esses pacientes, devendo ser analisado os hábitos alimentares desses enfermos, se o paciente leva um estilo de vida de forma adequada, se possuem refluxos gástricos, se utilizam próteses dentárias, analisar todos os fármacos que eles tomam para evitar possíveis efeitos adversos, analisar como é feito a higiene oral, e quantas vezes por dia é estabelecida esse processo de higienização (FERRAZ GA e LEITE ISG. 2016).

Um dos principais pontos que deve ser levado em consideração é o hálito do paciente, visto que ele pode ser um indicador de que algo não está correto, por essa razão, tem que ser analisado. O CD deve saber qual material está sendo utilizado para a limpeza da cavidade bucal, e qual técnica está sendo implantada, buscando sempre adaptar tanto os instrumentos como escovas, fio dental com o objetivo de melhorar e até mesmo facilitar na hora da higienização (SALAS MM, et al., 2015).

O nível de dependência do paciente por cuidadores sempre é analisado e observado pelos CD's, isso ocorre porque quando o paciente precisa do auxílio de algum parente ou cuidador para realizar essa higienização, precisa-se orienta-los de forma correta, de maneira particularizada, sendo modificada de um paciente para outro, tendo em consideração todos os empecilhos (SILVA RM, et al., 2018).

\section{Puérperas e recém-nascidos}

O processo de integração de cuidados imediatos tanto das mães quantos das crianças recém-nascidas é de extrema relevância pela Atenção Básica de saúde proposta pelo SUS, é recomendado que seja estabelecido visita em domicilio na primeira semana logo após a saída do bebê para a casa. Se a criança tiver nascido prematuramente ou possuir algum risco de vida, a equipe multiprofissional deve se fazer presente nas primeiras 72 horas no AD (BIZERRIL DO, et al., 2015). A AD ao que se refere a parte obstétrica e neonatal tem que possuir como atributos fundamentais a peculiaridades do processo humanitário, visto que é obrigação a prestação dos cuidados necessários ao que se refere à saúde e acolhimento da mulher e do bebê, evidenciando-os como usuários por direitos (SALAS MM, et al., 2015).

Silva RM, et al. (2018) relatam que a forma do atendimento tanto da mãe quanto do recém-nascido, devem apresentar atenção, focando-se na cavidade oral de ambos, possuindo como um dos principais fundamentos: correlacionar patologias orais e problemas que gerou resultados não tão benéficos na gravidez. 
Por exemplo, mortalidade pouco antes ou depois do parto, nascimento prematuro ou com peso abaixo do esperado e pré-eclâmpsia (Complicação potencialmente perigosa da gravidez, caracterizada por pressão arterial elevada), incentivar o aleitamento por meio de informes sobre sua relevância, reconhecer ocorrências de risco e direcionara-las a importância das práticas da higienização oral tanto delas quanto dos bebês.

\section{CONSIDERAÇÕES FINAIS}

$O A D$ estabelecido pela equipe multiprofissional dos quais incluem o $C D$ proporciona um ajustamento benéfico em que pode-se observar o processo de assistencialismo bastante humanizado principalmente aos usuários do SUS que não dispõem de um acesso adequado na porta de entrada ao serviço oferecido, Inúmeros grupos de pessoas apresentam necessidades do acompanhamento domiciliar porém pode ser observado que três grupos são destacados: os pacientes com PNEs, os acamados e Puérperas /Recémnascidos. À vista disto, os CD's nas visitas domiciliares encarrega-se de um fundamental papel, em que proporciona um conforto de maneira física e social dos pacientes que são incapacitados por diversas maneiras de irem até Unidade de atendimento, tendo como um dos principais objetivos estabelecer uma padronização de cuidados individualizados na cavidade oral, visto que a falta desses cuidados podem agravar as doenças sistêmicas que estabeleceram o quadro atual desses pacientes.

\section{REFERÊNCIAS}

1. BARATIERI T, MARCON SS. Longitudinalidade do cuidado: compreensão dos enfermeiros que atuam na estratégia saúde da família. Esc Anna Nery. 2011; 15(4):802-10.

2. BIZERRIL DO, et al. Papel do cirurgião-dentista nas visitas domiciliares: atenção em saúde bucal. Rev Bras Med Fam Comunidade. 2015;10(37)1-8.

3. BRASIL. Ministério da Saúde. Portaria MS/GM n 1.444, de 28 de dezembro de 2000. Diário Oficial da União, Brasília, DF, 29 dez. 2000, № 250E, Sec. 1, p.85.

4. BRASIL. Ministério da Saúde. Secretaria de Atenção à Saúde. Departamento de Atenção Básica. Caderno de Atenção Básica, no 17. Brasília: Ministério da Saúde; 2006.

5. CAMPOS CC, et al. Manual prático para o atendimento odontológico de pacientes com necessidades especiais. Goiânia: Universidade Federal de Goiás; 2009.

6. COELHO FLG, SAVASSI LCM. Aplicação da escala de risco familiar como instrumento de priorização das visitas domiciliares. Rev Bras Med FamComunidade 2016; 1(2):19-26.

7. DE-CARLI AD, et al. Visita domiciliar e cuidado domiciliar na Atenção Básica: um olhar sobre a saúde bucal. Saude Debate 2015; 39(105):441-450.

8. FERRAZ GA, LEITE ISG. Instrumentos de visita domiciliar: abordagem da odontologia na estratégia saúde da família. Rev APS 2016; 19(2):302-314.

9. KOBAYASHI HM, et al. Family risk as adjunct for organizing the demand for oral health service in the Family Health Strategy. Rev Odontol UNESP 2015; 44(2):85-91.

10. MENICUCCI TMG. O Sistema Único de Saúde, 20 anos: balanço e perspectivas. Cad Saude Publica 2009; 25(7):1620-1625.

11. MOURA MS, et al. Saúde bucal na Estratégia de Saúde da Família em um colegiado gestor regional do estado do Piauí. Cien Saude Colet 2013; 18(2):471-480.

12. PINTO VG. Planejamento. In: Saúde Bucal Coletiva. São Paulo: Editora Santos, 6. ed. Cap. 2. 2013; 8-30.

13. REIS WG, et al. O trabalho do Cirurgião-Dentista na Atenção Primária à Saúde: entre o prescrito e o real. Saude Debate 2015; 39(104):56-64.

14. REIS WG, et al. O trabalho do Cirurgião-Dentista na Atenção Primária à Saúde: entre o prescrito e o real. Saude Debate 2015; 39(104):56-64.

15. RODRIGUES FFL, et al. Relação entre conhecimento, atitude, escolaridade e tempo de doença em indivíduos com diabetes mellitus. Acta Paul Enferm. 2012; 25(2):284-90.

16. RODRIGUES FFL, et al. Relação entre conhecimento, atitude, escolaridade e tempo de doença em indivíduos com diabetes mellitus. Acta Paul Enferm. 2012; 25(2):284-90

17. SALAS MM, et al. Estimated prevalence of erosive tooth wear in permanent teeth of children and adolescents: an epidemiological systematic review and metaregression analysis. J Dent. 2015; 43(1): 42-50.

18. SILVA RM, et al. A visita domiciliar como prática pedagógica na formação em Odontologia. Rev ABENO 2017; $17(4): 87-98$.

19. SILVA RM, et al. Atuação da equipe de saúde bucal na atenção domiciliar na estratégia saúde da família: uma revisão integrativa. Rev. Ciência \& saúde 2018.

20. SILVA RM. Atenção à saúde bucal no domicílio no contexto da Estratégia Saúde da Família: reflexões a partir de uma revisão integrativa da literatura [monografia]. Florianópolis (SC): Universidade Federal de Santa Catarina; 2016.

21. SILVESTRE JAC, et al. Do Brasil em dentes ao Brasil sorridente: um resgate histórico das políticas públicas de saúde bucal no Brasil. Cadernos ESP 2013; 7(2):28-39. 